\title{
Potential Structural Obstacles to Effective Implementation of Neonatal Intensive Care Unit Rapid Response Teams
}

\section{Prithwijit Das, Tammy Pham, Laura Fletcher, Maguire Herriman and Ruth Milanaik}

Division of Developmental and Behavioral Pediatrics, Steven and Alexandra Cohen Children's Medical Center of New York, Lake Success, New York, USA

"Corresponding author: Ruth Milanaik, Division of Developmental and Behavioral Pediatrics, Steven and Alexandra Cohen Children's Medical Center of New York, Lake Success, New York, USA, Tel: (516) 633-7416; E-mail: rmilanai@northwell.edu

Received date: July 03, 2017; Accepted date: August 25, 2017; Published date: September 05, 2017

Copyright: @2017 Das P. This is an open-access article distributed under the terms of the Creative Commons Attribution License, which permits unrestricted use, distribution, and reproduction in any medium, provided the original author and source are credited.

\begin{abstract}
Background: In-hospital rapid response teams are critical to the prompt management of neonatal emergencies occurring in hospital delivery rooms. However, structural obstacles that exist within health care facilities may hinder the successful implementation of these teams. This study examined the prevalence of structural obstacles potentially impeding neonatal rapid response team movements between neonatal intensive care units and delivery rooms in hospitals with pediatric residency programs across the United States.
\end{abstract}

Methods: One resident from each participating pediatric residency program was sent an anonymous online questionnaire to record the total number of steps needed to walk the most direct route between the neonatal intensive care unit and delivery room of their health care facility. Residents also recorded the number of doors and elevators located along this route as well as the minimum amount of time required to open them. In a follow-up survey, residents reported whether elevator or security door overrides existed in the event of an emergency.

Results: One pediatric resident from each of 52 residency programs completed the questionnaire. On average, residents took 93 steps to get from the neonatal intensive care unit to the delivery room. At 30 hospitals (58\%), the neonatal intensive care unit and delivery room were on different floors, requiring residents to use stairs or elevators. Of the 18 residents who responded to the follow-up survey, 14 (78\%) reported either that there were no measures in place to expedite elevator or door travel or that they were not aware of any such measures. Between 30 and 60 seconds were spent opening doors or waiting for doors to open at six hospitals (12\%).

Conclusion: Structural obstacles in health care facilities may prevent units from being on the same floor, heightening the risk of injury for medical staff responding to neonatal emergencies, and increasing delays. Health care facility designs should take into consideration the impact of these obstacles in order to save valuable time and improve newborn outcomes.

Keywords: Neonatal intensive care unit; Rapid response team; Delivery room; Hospital; Preterm

\section{Introduction}

Neonatal emergencies can arise at any time around a baby's birth and during the subsequent in-hospital post-birth period. Preterm birth complications have been deemed the leading cause of mortality among children under the age of five [1,2]. Even when the infant is full term, the American Academy of Pediatrics estimates that about 1 in 10 newly born infants will require some resuscitation intervention [3]. Therefore, rapid response times of neonatal resuscitation teams responding to emergencies in delivery rooms are essential, potentially impacting the eventual outcomes of these infants $[4,5]$.

In-hospital rapid response teams are necessary for the successful treatment of neonatal emergencies [6]. Previous studies have evaluated efforts to improve the mobilization and performance of neonatal resuscitation teams. These studies highlight non-structural challenges to successful implementation such as breakdowns in communication and teamwork, protocol deviations, lack of confidence among team members, and difficulties in scheduling and equipment maintenance [7-11]. Physical obstacles such as staircases, elevators, automatic doors with lag time, and excessive distance may also impede rapid response teams and delay the management of emergency deliveries and birth complications, ultimately resulting in adverse maternal, fetal, and/or neonatal outcomes. However, no study to date has examined these structural obstacles present within the facility designs of hospitals that can potentially hinder movement and immediate response of neonatal intensive care unit (NICU) response teams. The purpose of this study is to evaluate the structural obstacles delaying the movement of rapid response teams between the NICU and delivery room in hospitals with pediatric residency programs in the United States.

\section{Methods}

The protocol for this study was approved by the Institutional Review Board of the Cohen Children's Medical Center of New York. Research personnel contacted chief residents and residency coordinators from all accredited pediatric residency programs in the United States via telephone, and a subsequent e-mail was sent with an anonymous, online questionnaire. Exactly one pediatric resident from each of the pediatric residency programs was asked to participate in the study in order to prevent overlap. Participating residents downloaded the free "Pacer-Pedometer" or "Noom Walk" application depending on their 
type of smartphone. Residents were then asked to walk the most direct route between the NICU and delivery room in their healthcare facility and record the number of steps taken using the pedometer application. They also recorded the number of doors opened throughout the route, the minimum time needed to open each door, and the number of stairwell and elevator trips taken. In a follow-up survey, the same pediatric residents were asked whether elevators and door systems were programmed with overrides that unlock the doors in the event of an emergency. From these responses, the research team quantified the structural obstacles and identified areas in need of improvement. Residents were given a ten-dollar restaurant gift card upon completion of the study.

\section{Results}

Pediatric residents from 52 pediatric residency programs responded to the study request (response rate $=25 \%$ ). Participants reported walking an average of 93 steps (approximately 240.25 feet) to get from the delivery room to the NICU using the most direct route in the healthcare facilities. Units at four hospitals (8\%) were separated by $\geq$ 200 steps, or approximately one-tenth of a mile (Figure 1).

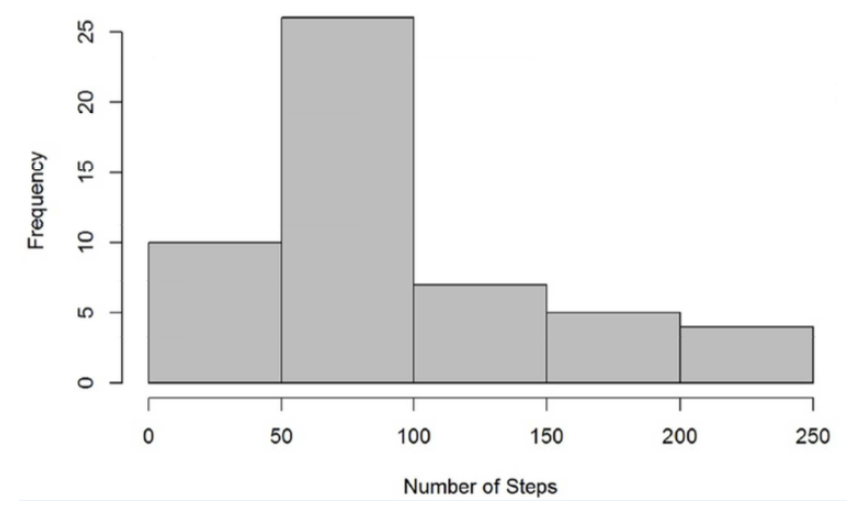

Figure 1: Histogram of the number of steps it takes pediatric residents to walk between the NICU and delivery rooms.

At 30 hospitals (58\%), participants noted that the NICU and delivery room were located on different floors, requiring residents to use stairs or elevators. Of the 18 residents who responded to the follow-up survey, 14 (78\%) reported either that there were no measures in place to expedite elevator or door travel or that they were not aware of any such measures. At six hospitals (12\%), participants reported that between $30 \mathrm{sec}$ and $60 \mathrm{sec}$ were spent just opening doors or waiting for doors to open (Figure 2).

\section{Discussion}

Immediate response to delivery room and NICU emergencies is vital to achieving successful neonatal outcomes. Rapid response teams can play a critical role in reducing mortality and code rates [12]. Yet research on improving response times of neonatal rapid response teams is limited. In 2008, Villamaria et al. reported that $14 \%$ of Code Blue Teams identified issues with crowded elevators and stairs in a healthcare facility, recognizing these as barriers to effective response and decision-making [13]. Infants without apparent risk factors may without warning require special assistance and treatment. Thus, healthcare facilities must ensure that protocols are in place that allow for the rapid mobilization of health professionals who are prepared to manage these emergencies. All efforts should be made to decrease structural obstacles that hinder prompt response. Units on different floors require responders to either rush up or down stairs (increasing the risk for injury), or wait for an elevator (increasing delays) [14].

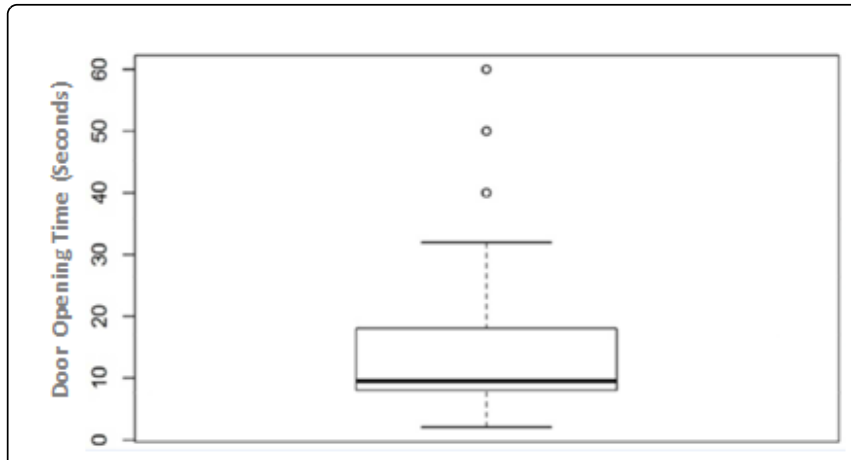

Figure 2: Boxplot of the distribution of time pediatric residents spent opening doors and waiting for doors to open when walking the most direct route between the NICU and delivery room.

A study by Terrin et al. noted the potential for significant reductions in preterm neonate morbidity in facilities where the delivery room and NICU were directly connected as opposed to being on separate floors [15]. Healthcare facilities without programmed override measures for door systems further contribute to unnecessary delay in initiation of emergency services. Considerations should also be made to designing automatic doors at the top and bottom of stairwells or assigning staff to hold doors open to reduce delays in response. Even in situations where building layout prevents units from being on the same floor, efforts to streamline travel between delivery rooms and the NICU such as installing rapidly opening doors in the hallways, can save precious seconds during an emergency and potentially reduce neonatal morbidity and mortality. Although existing hospitals are constrained by current infrastructure, the findings of this study suggest that future arrangement of hospital units should consider the functional relationships between hospital areas to ensure safe and efficient travel between the delivery room and the NICU. Considerations for future facility design and organization should ensure that NICUs are in close proximity to delivery rooms.

While there were several limitations to this study, our findings are nevertheless important. All hospitals with NICUs should consider the structural obstacles present in their facilities and what could be done to address them and improve response times. One limitation was that the study resulted in a relatively low response rate, potentially introducing selection bias and limiting the generalizability of these findings. Yet, our relatively low participation rate may underscore the extent of the structural obstacles present in healthcare facilities around the country. Future studies should continue investigating the prevalence of various structural obstacles in healthcare facilities that can delay rapid response systems.

\section{Conclusions}

This study highlights the prevalence of significant structural obstacles reported by pediatric residents between delivery rooms and the neonatal intensive care units of healthcare facilities. Fundamental 
Citation: Das P, Pham T, Fletcher L, Herriman M, Milanaik R (2017) Potential Structural Obstacles to Effective Implementation of Neonatal Intensive Care Unit Rapid Response Teams. Clinics Mother Child Health 14: 269. doi:10.4172/2090-7214.1000269

Page 3 of 3

changes to healthcare facility organization and design are necessary to address the serious threats that these obstacles pose to the prompt management of neonatal emergencies and to improve newborn outcomes.

\section{References}

1. World Health Organization. Preterm birth. World Health Organization.

2. Blencowe H, Cousens S, Oestergaard MZ, Chou D, et al. (2012) National, regional, and worldwide estimates of preterm birth rates in the year 2010 with time trends since 1990 for selected countries: a systematic analysis and implications. Lancet 379: 2162-2172.

3. Keenan WJ, Udaeta E, López M (2009) Delivery and Immediate Neonatal Care. In: Berman S, editors. Pediatric Education in Disasters Manual. American Academy of Pediatrics. Buenos Aires, Argentina 2009. pp: 217-237.

4. Fowlie PW, McGuire W (2004) ABC of preterm birth: Immediate care of the preterm infant. BMJ 329: 845-848.

5. Wyckoff MH, Aziz K, Escobedo MB, Kapadia VS, Kattwinkel J, et al. (2015) Part 13: neonatal resuscitation. Circulation 132: 543-560.

6. Kilday D, Spiva L, Barnett J, Parker C, Hart P (2013) The effectiveness of combined training modalities on neonatal rapid response teams. Clin Simul Nurs 9: 249-256.

7. Bailey C, Kattwinkel J (1990) Establishing a neonatal resuscitation team in community hospitals. J Perinatol 10: 294-300.
8. Carbine DN, Finer NN, Knodel E, Rich W (2000) Video recording as a means of evaluating neonatal resuscitation performance. Pediatrics 106: 654-658.

9. Finer NN, Rich W (2002) Neonatal resuscitation: toward improved performance. Resuscitation 53: 47-51.

10. Thomas EJ, Taggart B, Crandell S, Lasky RE, Williams AL, et al. (2007) Teaching teamwork during the Neonatal Resuscitation Program: A randomized trial. J Perinatol 27: 409-414.

11. Thomas EJ, Williams AL, Reichman EF, Lasky RE, Crandell S, et al. (2010) Team training in the neonatal resuscitation program for interns: teamwork and quality of resuscitations. Pediatrics 125: 539-546.

12. Sharek PJ, Parast LM, Leong K, Coombs J, Earnest K, et al. (2007) Effect of a rapid response team on hospital-wide mortality and code rates outside the ICU in a children's hospital. Jama 298: 2267-2274.

13. Villamaria FJ, Pliego JF, Wehbe-Janek H, Coker N, Rajab $\mathrm{MH}$, et al. (2008) Using simulation to orient code blue teams to a new hospital facility. Simul Healthc 3: 209-216.

14. Benin AL, Borgstrom CP, Jenq GY, Roumanis SA, Horwitz LI (2012) Defining impact of a rapid response team: qualitative study with nurses, physicians and hospital administrators. Postgrad Med J 88: 575-582.

15. Terrin G, Conte F, Scipione A, Aleandri V, Di Chiara M, et al. (2016) New architectural design of delivery room reduces morbidity in preterm neonates: a prospective cohort study. BMC Pregnancy Childbirth 16: 63. 